\title{
PRONTUÁRIO ELETRÔNICO DO PACIENTE: AVALIAÇÃO DE USABILIDADE PELA EQUIPE DE ENFERMAGEM*
}

Janaína Verônica Lahm¹, Deborah Ribeiro Carvalho²

'Enfermeira. Mestre em Tecnologia em Saúde. Pontifícia Universidade Católica do Paraná. Toledo-PR- Brasil.

${ }^{2}$ Analista de Sistemas. Doutora em Informática. Pontifícia Universidade Católica do Paraná. Toledo-PR- Brasil.

RESUMO: O objetivo deste trabalho foi avaliar a usabilidade e as dificuldades encontradas por 99 profissionais de enfermagem no manuseio de prontuário eletrônico do paciente. Pesquisa exploratória quantitativa a partir da coleta de dados no período de julho a novembro de 2013 . Os resultados demostram que $71 \%$ dos auxiliares/ técnicos e $70 \%$ dos enfermeiros não receberam treinamento específico; sendo que $56 \%$ da equipe, que respondeu não ter recebido treinamento, apresenta dificuldade no uso. Dentre as características avaliadas de usabilidade do prontuário eletrônico do paciente destacam-se positivamente a adequação à tarefa e negativamente à adequação ao aprendizado. Portanto, o sistema avaliado, apesar dos avanços advindos, ainda se apresenta complexo para o usuário que não recebeu treinamento, apesar de possuir interface consistente e interativa.

DESCRITORES: Enfermagem; Sistemas computadorizados de registros médicos; Avaliação.

\section{ELECTRONIC HEALTH RECORDS: EVALUATION OF USABILITY BY THE NURSING TEAM}

\begin{abstract}
This work aimed to evaluate the usability and the difficulties met by 99 nursing professionals using electronic health records. It is exploratory quantitative research, based on data collected in July - November 2013. The results show that $71 \%$ of the auxiliary nurses/ nursing technicians and $70 \%$ of the nurses had not received specific training; $56 \%$ of the team, who stated that they had not received training experienced difficulty in using the system. Among the characteristics of usability of the electronic health record evaluated, suitability to the task stood out positively, while suitability to learning stood out negatively. The system evaluated, therefore, in spite of the advances arising from it, is still presented as complex to the user who has not received training, in spite of its having a consistent and interactive interface.
\end{abstract}

DESCRIPTORS: Nursing; Computerized medical record systems; Evaluation.

\section{PRONTUARIO ELECTRÓNICO DEL PACIENTE: EVALUACIÓN DE USABILIDAD POR EL EQUIPO DE ENFERMERÍA}

RESUMEN: El objetivo de este trabajo fue evaluar la usabilidad y las dificultades halladas por 99 profesionales de enfermería en el manoseo de prontuario electrónico del paciente. Investigación exploratoria cuantitativa hecha por medio de la obtención de datos en el periodo de julio a noviembre de 2013. Los resultados apuntan que $71 \%$ de los auxiliares/técnicos y $70 \%$ de los enfermeros no tuvieron entrenamiento específico; siendo que $56 \%$ del equipo, que contestó no tener entrenamiento, presenta dificultad en el uso. Entre las características evaluadas de usabilidad del prontuario electrónico del paciente se destacan positivamente la adecuación a la tarea y, negativamente, la adecuación al aprendizaje. Por lo tanto, el sistema evaluado, a pesar de los avances, todavía se presenta complejo para el usuario que no tuvo entrenamiento, a pesar de poseir interfaz consistente e interactiva.

DESCRIPTORES: Enfermería; Sistemas computadorizados de registros médicos; Evaluación.

*Artigo extraído da dissertação intitulada "Prontuário eletrônico do paciente: avaliação de usabilidade pela equipe de enfermagem". Pontifícia Universidade Católica, 2014. 


\section{INTRODUÇÃO}

Os sistemas de informações e registros eletrônicos surgem nos hospitais e redes de saúde em meados de 1960, objetivando facilitar os registros da atenção, evoluindo para uma sistematização não apenas de dados gerenciais, mas também daqueles referentes à atenção aos pacientes $^{(1)}$.

O Prontuário Eletrônico do Paciente (PEP) registra dados de saúde e doença indispensáveis para a comunicação entre a equipe multidisciplinar e o paciente, garantindo não apenas o histórico do processo, mas também a segurança e a gestão das organizações de saúde ${ }^{(2)}$. Assim sendo, o PEP deve ser entendido como "a estrutura eletrônica para manutenção de informação sobre o estado de saúde e o cuidado recebido por um indivíduo durante todo seu tempo de vida"(3:213).

A enfermagem é parte essencial no desenvolvimento e na operacionalização do PEP, por ter participação ativa nos registros de saúde dos pacientes a partir de funcionalidades específicas $^{(4)}$.

O PEP constitui uma alternativa utilizada pela enfermagem para o registro de suas atividades de assistência, sendo contemplado na Lei do Exercício Profissional da Enfermagem - COFEN( ${ }^{(5)}$. $\mathrm{O}$ artigo $8^{\circ}$ aponta a exigência da utilização de recursos computacionais para o planejamento das ações, conforme as competências de cada grupo profissional da enfermagem, bem como o preparo necessário para a utilização deste recurso.

Os recursos computacionais têm sido utilizados como uma alternativa no apoio ao desenvolvimento do Processo de Enfermagem $(\mathrm{PE})$, oportunizando integrá-lo em uma estrutura lógica de dados, informação e conhecimento para a tomada de decisão do cuidado sistematizado(6).

A integração do PE ao PEP constitui um dos benefícios advindos da informatização da assistência de enfermagem, selecionando e inserindo os dados do paciente, conforme a estrutura de funcionamento implementada no software, disponibilizando sugestões de diagnósticos, permitindo evidenciar as intervenções a serem implementadas pela enfermagem, resultando em prescrições para o cuidado $^{(7)}$.

A avaliação da qualidade do Sistema de Informação (SI) é definida como a qualidade em uso, visão da condição do produto, utilizado em ambiente e contexto específicos, medindo o quanto os usuários podem atingir seus objetivos ${ }^{(8)}$.

Uma das estratégias para avaliar a qualidade de produtos de softwares e SI é a usabilidade. Para Cybis a usabilidade é definida como a qualidade que caracteriza o uso dos programas e aplicações e para Nielsen refere-se à rapidez com que os usuários aprendem a usar alguma coisa, a eficiência em usá-la, a lembrança dos comandos e se gostam de usar algum recurso ${ }^{(9-10)}$.

$\mathrm{Na}$ avaliação da usabilidade os usuários são observados e entrevistados, obtendo assim o feedback sobre o produto em questão. Para tal, podem ser adotados testes e questionários com participação direta ou indireta destes usuários ${ }^{(11-12)}$ que determinam a capacidade e o envolvimento das pessoas que fazem o uso de ferramentas computacionais, possibilitando determinar o uso em tempo hábil e com altos níveis de satisfação ${ }^{(13)}$.

Diante deste contexto a questão que direciona esta pesquisa é: "é possível, a partir da avaliação do prontuário eletrônico do paciente pelos profissionais da enfermagem, identificar problemas de usabilidade?".

Portanto, esta pesquisa objetiva avaliar a qualidade, a partir de critérios da usabilidade, e as dificuldades dos profissionais de enfermagem na utilização de um prontuário eletrônico do paciente.

\section{MÉTODO}

Este estudo se caracteriza como descritivo, exploratório com abordagem quantitativa, tendo como objeto de estudo o módulo do PEP específico para a utilização da equipe de enfermagem que está integrado ao software Tasy ${ }^{\circledR}$. Este módulo conta com diversas funcionalidades, sendo selecionadas as janelas "Sistematização da Assistência de Enfermagem (SAE)" "e Evolução / Anamnese", por serem de acesso comum e obrigatório por todas as classes de profissionais da enfermagem.

A pesquisa, aprovada pelo Comitê de Ética em Pesquisa sob número 290.833/2013, foi desenvolvida em um hospital da região Sul do Brasil, no período de julho a novembro de 2013. Durante o período de realização da pesquisa, 
estavam trabalhando no hospital 361 profissionais de enfermagem, considerando as três classes. Para operacionalizar a pesquisa foi calculado o tamanho da amostra, considerando a população de profissionais no momento da avaliação (população finita) e uma estimativa inicial de 50\% para o percentual de interesse, nível de confiança de $95 \%$ e margem de erro máxima de $9 \%$, o que definiu por pelo menos 99 profissionais, sendo 20 enfermeiros, 79 técnicos e auxiliares de enfermagem.

Como critério de inclusão foi adotado a seleção aleatória simples, sorteando 99 profissionais das unidades de atendimento, com o objetivo de apresentar a mesma probabilidade entre aqueles que trabalhavam na instituição por mais de um ano, quando da realização da pesquisa. Em situações de negativas foi realizada nova seleção. A seleção aleatória se justifica para evitar qualquer viés durante a etapa de coleta de dados.

$\mathrm{Na}$ operacionalização da coleta dos dados, foram construídos dois instrumentos, avaliados, inicialmente, em um teste piloto envolvendo 10 profissionais. Os instrumentos foram: (a) caracterização do perfil e experiência em informática composto por sete variáveis (número de horas semanais em que utiliza o computador, realização de curso básico em informática, habilidades para edição, local mais frequente de utilização do computador, ferramenta mais utilizada, dificuldades em utilizar o PEP e internet) $\mathrm{e}^{(\mathrm{b})}$ teste de usabilidade composto por dez variáveis (adequação à tarefa, autodescrição, controlabilidade, conformidade com as expectativas do usuário, tolerância a erros, adequação à individualização e adequação ao aprendizado, quantificação da interrupção do ambiente, tempo de execução do teste, apoio para completar o teste e nível de tensão).

Todos os instrumentos seguiram o modelo da ISONORM 9241/10 e seus princípios de diálogo que avalia a qualidade de software e considera questões sobre usabilidade na visão do usuário, bem como suas características ergonômicas, sendo eles: a) adequação à tarefa quando o sistema auxilia o usuário em uma conclusão efetiva e eficiente da tarefa; b) autodescrição é quando cada passo do diálogo é imediatamente compreensível por meio de resposta do sistema ou é explicado, sob demanda, ao usuário; c) controlabilidade é quando o usuário pode iniciar e controlar a direção e o ritmo da interação até que o objetivo tenha sido atingido; d) conformidade com as expectativas do usuário quando o sistema é consistente e corresponde às características do usuário; e) tolerância ao erro é quando o resultado esperado pode ser obtido com pouca ou nenhuma ação corretiva do usuário; f) adequação à individualização quando o sistema é capaz de individualização e a interface pode ser modificada para se adequar às necessidades da tarefa, preferências individuais e habilidades do usuário; g) adequação ao aprendizado é quando o sistema apóia e guia o usuário no aprendizado para usá-lo ${ }^{(14)}$.

O teste foi realizado, individualmente, com cada participante no local de trabalho, quando cada participante completava as tarefas em conformidade com a situação simulada, com a finalidade de avaliar a usabilidade do produto.

A avaliação dos resultados do teste piloto, bem como da pesquisa propriamente, ocorreu mediante a leitura das respostas, demarcação das respostas relevantes com contribuição de diferentes componentes do contexto e aplicação da escala de concordância de Likert, com respostas fechadas dispostas em uma escala de peso que variou de 2 a -2 , indo de "concordo plenamente" (peso 2); "concordo" (peso 1); "talvez" (peso 0); "discordo" (peso -1) e "discordo plenamente" (peso -2), obtendo-se portanto, valores máximos para cada questão entre -40 a 40 pontos (classe enfermeiro) e -158 a 158 pontos (classe técnico de enfermagem).

Os resultados da pesquisa foram sistematizados em planilha eletrônica, processados no pacote estatístico SPSS 13.0. Foram analisados os resultados com nível de significância convencionado de $\mathrm{p}<\mathrm{ou}=0,05$.

\section{RESULTADOS}

A partir dos resultados $52 \%(n=41)$ dos auxiliares e técnicos tinham entre 40 a 59 anos e $70 \%(n=14)$ dos enfermeiros entre 20 e 39 anos. Observou também que $60 \%(\mathrm{n}=47)$ dos auxiliar e técnico de enfermagem e 65\% $(n=13)$ dos enfermeiros realizaram curso básico em informática, porém $71 \%(n=56)$ dos auxiliares e técnicos de enfermagem e 35\% $(n=7)$ dos enfermeiros afirmaram ter dificuldades em usar 
ferramentas de edição de texto.

Em relação ao treinamento para usar o PEP percebeu-se que $71 \%(n=56)$ dos auxiliares e técnicos e $70 \%(n=14)$ dos enfermeiros atestaram não ter recebido. Vale destacar que $56 \%(n=39)$ dos que atestaram não ter recebido treinamento apresentaram dificuldades na utilização do PEP $(p=0,015)$. Quanto ao grau de escolaridade, aqueles que possuíam somente ensino médio completo $63 \%(n=20)$ afirmaram ter dificuldades; em contrapartida, 69\% ( $n=22)$ dos que possuíam superior completo atestaram não ter dificuldades $(p=0,043)$.

Dentre os que responderam ter dificuldades na utilização do PEP 41\% (n=41) demandou apoio durante a realização do teste, sendo que para aqueles que atestaram não ter dificuldade, 32\% $(n=31)$ também solicitou apoio $(p=0,003)$. Vale destacar que, ao todo, $73 \%(\mathrm{n}=72)$ dos profissionais participantes da pesquisa demandaram apoio (Tabela 1).

Tabela 1 - Resultados do perfil profissional e dificuldades em usar o PEP. Cascavel-PR, 2013

\begin{tabular}{lccc}
\hline Variáveis & \multicolumn{2}{c}{ Enfermeiros } & Técnicos/auxiliares de enfermagem \\
\hline Faixa etária & $70 \%(20$ e 39 anos $)$ & $52 \%$ (40 a 59 anos $)$ \\
\hline Curso básico em informática & \multicolumn{2}{c}{$65 \%$} & $60 \%$ \\
\hline Dificuldades em edição de texto & \multicolumn{2}{c}{$35 \%$} & $71 \%$ \\
\hline Não ter treinamento & \multicolumn{2}{c}{$70 \%$} & Não \\
\hline Dificuldades em usar o PEP & Sim & $44 \%$ & Nível de significância \\
\hline Não ter treinamento & $56 \%$ & $69 \%$ & $\mathrm{p}=0,015$ \\
\hline Ensino superior & - & - & $\mathrm{p}=0,043$ \\
\hline Ensino médio & $63 \%$ & $32 \%$ & $\mathrm{p}=0,043$ \\
\hline Apoio para o teste de usabilidade & $41 \%$ & & $\mathrm{p}=0,003$ \\
\hline
\end{tabular}

Quanto à avaliação de usabilidade realizada pelos enfermeiros, é possível identificar somatórias positivas na escala de concordância de Likert de 26 e 28 pontos (somatória máxima positiva é de 40 pontos) para os itens avaliação adequação à tarefa e conformidade com a expectativa do usuário e negativos -13 e-15 pontos (somatória máxima negativa é de -40 pontos) para controlabilidade e adequação ao aprendizado respectivamente. Na avaliação de usabilidade realizada pelos auxiliares/técnicos foram encontrados resultados positivos 106 e 107 pontos (somatória máxima positiva é de 158 pontos) para adequação à tarefa e tolerância ao erro e negativos -35 e -57 pontos (somatória máxima negativa é de -158 pontos) para conformidade com a expectativa do usuário e adequação ao aprendizado (Tabela 2).

Tabela 2 - Resultados da avaliação de usabilidade por classe profissional. Cascavel-PR, 2013

\begin{tabular}{|c|c|c|c|c|c|c|}
\hline \multirow{3}{*}{$\begin{array}{l}\text { Princípios } \\
\text { ISONORM } 9241\end{array}$} & \multirow{3}{*}{ Questões } & \multicolumn{5}{|c|}{ Classe } \\
\hline & & \multicolumn{2}{|c|}{ Enfermeiro } & \multicolumn{2}{|c|}{$\begin{array}{c}\text { Técnico e Auxiliar de } \\
\text { Enfermagem }\end{array}$} & \multirow{2}{*}{$\begin{array}{c}\text { Falas } \\
\text { negativas }\end{array}$} \\
\hline & & $\begin{array}{c}\text { Escala de } \\
\text { Likert }[-40 / 40]\end{array}$ & Concordo \% & $\begin{array}{c}\text { Escala de Likert } \\
{[-158 / 158]}\end{array}$ & Concordo \% & \\
\hline Adequação à tarefa & $\begin{array}{l}\text { Q1 } \\
\text { Q7 }\end{array}$ & 26 & $95 \%$ & 106 & $94 \%$ & - \\
\hline Tolerância ao erro & Q3 & - & - & 107 & $96 \%$ & - \\
\hline $\begin{array}{l}\text { Conformidade } \\
\text { com a expectativa }\end{array}$ & $\begin{array}{l}\text { Q3 } \\
\text { Q13 }\end{array}$ & 28 & $90 \%$ & -35 & $56 \%$ & $\begin{array}{l}\text { E52: Eu não } \\
\text { sei, porque a } \\
\text { gente não teve } \\
\text { treinamento. }\end{array}$ \\
\hline $\begin{array}{l}\text { Adequação ao } \\
\text { aprendizado }\end{array}$ & $\begin{array}{l}\text { Q9 } \\
\text { Q15 }\end{array}$ & -15 & $60 \%$ & -57 & $72 \%$ & $\begin{array}{l}\text { E13: Eu tenho } \\
\text { dificuldade, é } \\
\text { difícil. }\end{array}$ \\
\hline Controlabilidade & Q11 & -13 & $70 \%$ & - & - & $\begin{array}{l}\text { E10: Eu acho } \\
\text { que falta } \\
\text { treinamento. }\end{array}$ \\
\hline
\end{tabular}


Portanto, o PEP foi avaliado positivamente por ambas as classes quanto à adequação à tarefa e negativamente quanto à adequação ao aprendizado.

A avaliação sobre possíveis diferenças entre os profissionais dos diversos setores não apresentou significância.

\section{DISCUSSÃO}

Com os resultados obtidos foi possível avaliar a usabilidade do PEP a partir das variáveis selecionadas e relacionadas à equipe de enfermagem.

Quanto à idade dos profissionais pesquisados houve a predominância de sujeitos jovens na classe enfermeiro, tendo em vista a característica de uma nova geração de indivíduos que iniciam suas vidas profissionais na Era da Informação ${ }^{(15)}$.

Vale ressaltar que toda a equipe de enfermagem utilizava o PEP, porém para a inserção de dados utilizam funcionalidades distintas de acordo com a classe à qual pertenciam.

Os fatores que influenciaram no uso do PEP sem dificuldades foram a capacitação e o grau de escolaridade, pois a maioria dos profissionais que possuía somente ensino médio e não recebeu capacitação atestou ter dificuldades na utilização do PEP.

Quanto a ter realizado curso básico de computação, os resultados refletem o posicionamento da enfermagem frente à realidade de um mundo informatizado, bem como a busca pelo aperfeiçoamento. Entretanto, profissionais declararam ter dificuldade no manuseio de ferramentas básicas, como editor de texto, fato que pode prejudicar o desempenho das atividades profissionais, considerando o contexto da crescente dependência da informática.

Este fato é importante, pois os recursos computacionais podem promover a melhoria da qualidade do cuidado da enfermagem, considerando a redução do tempo necessário para efetivar os registros, bem como estimular o desenvolvimento do pensamento crítico e do raciocínio investigativo dos enfermeiros, favorecendo a discussão clínica entre a equipe multidisciplinar e busca de evidências científicas ${ }^{(16)}$.

Assim, a apropriação de recursos tecnológicos permite que a enfermagem despenda um tempo maior na assistência direta ao paciente, otimizando o processo de trabalho. Deste modo, os enfermeiros devem estar atentos para o desenvolvimento de competências e habilidades para a utilização de tecnologias computacionais ${ }^{(17)}$.

Quanto às dificuldades em utilizar o PEP, os resultados reforçam uma preocupação, pois este recurso está sendo inserido diariamente na rotina de serviços de saúde e muitos enfermeiros ainda não se sentem seguros para a sua utilização, necessitam de auxílio para acessar comandos básicos e de rotina do PEP, situação esta que pode não contribuir para as atividades de assistência ao paciente e pela busca da qualidade e eficácia da atenção em saúde.

Nesta pesquisa a necessidade de auxílio para trabalhar com recursos informacionais adveio do não treinamento sistemático, pois aproximadamente $70 \%$ dos profissionais entrevistados declaram não ter treinamento, evidenciando uma fragilidade. Uma pesquisa aponta a "existência de uma íntima relação entre treinamento para utilização do PEP e sua utilização sem dificuldades pelos enfermeiros" ${ }^{\prime \prime 15: 73)}$. Em outra pesquisa foi constatado que os enfermeiros não possuíam conhecimento suficiente para o uso do PEP e para aplicação do PE, o que pode prejudicar a inserção de dados da assistência e a qualidade da atenção ${ }^{(18)}$.

A despeito da preocupação de que a modelagem do PEP seja a mais adequada possível ao seu uso, se os profissionais não forem capacitados podem ocorrer falhas na comunicação multiprofissional e prejudicar a assistência ao paciente. A não capacitação impede que uma das vantagens da adoção do PEP seja atingida, maior e melhor comunicação entre os membros da equipe de saúde, resultando na melhoria da qualidade do atendimento prestado e possibilitando geração conhecimento e constante inovação ${ }^{(1)}$.

Portanto, o PEP para ser considerado um recurso tecnológico eficiente deve atender a requisitos e ser objeto de treinamento. Também, deve ser intuitivo facilitando seu uso, facilitando assim o treinamento, que deve ser gradual de acordo com a implantação do sistema ${ }^{(19-20)}$.

Na avaliação de usabilidade, o PEP apresentou pontos positivos quanto à adequação à tarefa, conformidade com a expectativa do usuário e tolerância ao erro e pontos negativos quanto à controlabilidade e adequação ao aprendizado. 
Estes resultados apontam que o uso eficiente do PEP também está relacionado com a satisfação e a aceitação do sistema, bem como com o tempo necessário para o registro dos dados A presença de eventos alerta estão diretamente relacionados à melhora da assistência ao paciente, orientando a sequência do raciocínio lógico a partir da sistematização da avaliação clínica e do PE(20-21).

É importante destacar que a linguagem adotada no SI de enfermagem é essencial para o entendimento e a descrição dos cuidados, devendo ser classificada, apoiando na tomada de decisão para as ações a serem tomadas em relação ao paciente de forma global ${ }^{(22)}$.

Quanto aos itens que resultaram em avaliação negativa, os técnicos de enfermagem destacaram adequação à tarefa, controlabilidade e conformidade com a expectativa do usuário, pois não tinham domínio para seguir com as tarefas propostas, necessitando auxílio da pesquisadora.

Fica clara a necessidade do usuário em buscar conhecimento básico em informática e receber treinamento adequado para poder utilizar com eficiência o sistema PEP estudado.

\section{CONSIDERAÇÕES FINAIS}

Este trabalho avaliou o perfil dos profissionais de enfermagem, a usabilidade e a dificuldade destes profissionais na utilização do PEP.

Tais dificuldades estão relacionadas à falta de conhecimento para o manuseio do PEP e comandos específicos de adequação a tarefa que facilitam o seu uso.

A complexidade do sistema também pode contribuir para ampliar a dificuldade de seu manuseio, bem como a falta de conhecimento básico em informática.

Ao discorrer sobre PEP deve-se salientar que sua implantação e sua utilização envolvem não apenas a troca do papel pelo meio eletrônico, mas mudanças de velhos hábitos, rotinas e aquisição de novos conhecimentos, os quais podem ser dificultados por uma atitude de resistência por parte dos usuários.

Os resultados desta pesquisa poderão servir como suporte aos demais hospitais públicos ou privados que utilizam o PEP, que tenham interesse em replicar a pesquisa para garantir a qualidade dos dados e cuidados aos pacientes. Também, poderá contribuir para pesquisas relacionadas à avaliação de qualidade e capacitação de usuários de PEP.

\section{REFERÊNCIAS}

1. Molina LG, Lunardelli RSA. O prontuário do paciente e os pressupostos arquivísticos: estreitas e profícuas interlocuções. Info [Internet] 2010; 15(1). [acesso em 11 out 2012]. Disponível: http://www.uel.br/revistas/ uel/index.php/informacao/article/view/4764

2. Pinto VB. Prontuário eletrônico do paciente: documento técnico de informação e comunicação do domínio da saúde. Rev Eletrônica [Internet] 2006; 11(21). [acesso em 18 mai 2012]. Disponível: http://www.periodicos.ufsc.br/index.php/eb/article/ view/1518-2924.2006v11n21p34

3. Massad E, Marin HF, Azevedo Neto RS, editores. O prontuário eletrônico do paciente na assistência, informação e conhecimento médico. [Internet] 2003 [acesso em 20 out 2011]. Disponível: http://www.sbis. org.br/site/arquivos/prontuario.pdf

4. Leea M, Delaney C, Moorhead DS. Building a personal health record from a nursing perspective. Inter J $M$ Info [Internet] 2007. [cited 02 ago 2012]. Disponível: http:// www.ncbi.nlm.nih.gov/pubmed/17616432

5. Conselho Federal de Enfermagem. Decreto n. 94.406/87 regulamenta a Lei $n^{\circ} 7.498$, de 25 junho 1986. Dispõe sobre o exercício da enfermagem, e dá outras previdências. [Internet] 1986. [acesso em 28 mai 2012]. Disponível: http://www.planalto.gov.br/ ccivil_03/decreto/1980-1989/D94406.htm

6. Dal Sasso GTM, Barra DCC, Paese F, Almeida SRW, Rios GC, Marinho M, et al. Processo de enfermagem informatizado: metodologia para associação da avaliação clínica, diagnósticos, intervenções e resultados. Rev. Esc. Enferm. USP. [Internet] 2013;47(1). [acesso em 13 jan 2014]. Disponível: http://dx.doi. org/10.1590/S0080-62342013000100031

7. Palomares MLE, Marques IR. Contribuições dos sistemas computacionais na implantação da sistematização da assistência de enfermagem. JHI [Internet] 2010; 2(3):78-82. [acesso em 05 abr 2013]. Disponível: http:// www.jhi-sbis.saude.ws/ojs-jhi/index.php/jhi-sbis/issue/ view/12

8. Associação Brasileira de Normas Técnicas - ABNT. NBR ISO/IEC 9126-1: engenharia de software qualidade de produto. Rio de Janeiro: ABNT; [Internet] 2003. [acesso em 07 jan 2013]. Disponível: http:// www.abntcatalogo.com.br/norma.aspx? ID =002815

9. Cybis W. Ergonomia e Usabilidade: conhecimentos, métodos e aplicações. São Paulo: Novatec Editora; 2007. 
10. Nielsen J. Loranger $H$. Usabilidade na web. Trad. de Edson Furmankiewicz e Carlos Schafranski. [Internet] Rio de Janeiro: Campus; 2007. [acesso em 11 jan 2014]. Disponível: http://books.google.com.br/books?hl=ptBR\&l $r=\& i d=5 h h F q \times 9 T M t Y C \& o i=f n d \& p g=P R 14 \& d q=$ nielsen+usabilidade \&ots $=20 i c$ Ahybof $\&$ sig $=d T n Y Y g D X$ evYSIGf_3D5GoqrHAw8\#v=onepage\&q=nielsen $\% 20$ usabilidade \&f=false

11. Santos RLG. Usabilidade e métodos de avaliação de usabilidade de interfaces web. Rio de Janeiro, Brasil 2000. [acesso em 11 out 2012]. Disponível: http://www.academia.edu/6883950/ USABILIDADE_E_M\%C3\%89TODOS_DE_ AVALIA\%C3\%87\%C3\%830_DE_USABILIDADE_ DE_INTERFACES_WEB

12. Ryan P, Pumilia NJ, Henak B, Chang T. Development and performance usability testing of a theory based, computerized, Tailored intervention. Comput Inform Nurs. [Internet] 2009; 27(5). [acesso em 26 set 2012]. Disponível: http://www.ncbi.nlm.nih.gov/ pubmed/19726922

13. Orth AI. Interface homem máquina. Porto Alegre: Editora AIO; 2005.

14. Associação Brasileira de Normas Técnicas. NBR 924111: requisitos ergonômicos para trabalho de escritórios com computadores parte 11 - orientações sobre usabilidade. Rio de Janeiro: ABNT; [Internet] 2002. [acesso em 27 jan 2012]. Disponível: http://www.inf. ufsc.br/ cybis/pg2003/iso9241-11F2.pdf

15. Florencio TF. Prontuário eletrônico do paciente: implicações para a assistência de enfermagem [dissertação]. Rio de Janeiro (RJ): Universidade Federal do Estado do Rio de Janeiro. [Internet] 2010; 149p [acesso em 26 mai 2012]. Disponível: http://www2.unirio.br/unirio/ccbs/ppgenf/arquivos/ dissertacoes-arquivo/dissertacoes-2010/tatianefernandes-florencio

16. Almeida DM. Sistematização da assistência de enfermagem informatizada em unidade de cuidado semi-intensivo [dissertação]. Bauru (SP): Universidade de São Paulo. [Internet] 2011; [acesso em 13 jan 2014]. Disponível: http://www.teses.usp.br/teses/ disponiveis/61/61132/tde-24052011-142633/pt-br.php

17. Cruz NS, Soares DKS, Bernardes A, Gabriel CS, Pereira MCA, Évora YDM. A competência técnica em informática de alunos de enfermagem. Rev. Esc. Enferm. USP. [Internet] 2011; 45(3) [acesso em 13 jan 2014]. Disponível: http://www.scielo.br/scielo.php?script=sci_ arttext\&pid=S0080-62342011000700009

18. Costa CGA. Desenvolvimento e avaliação tecnológica de um sistema de prontuário eletrônico do paciente, baseado nos paradigmas da World Wide Web e da engenharia de software [dissertação]. Campinas (SP): Universidade Estadual de Campinas; 2001.
19. Silva ML. Manual de Certificação para Sistemas de Registro Eletrônico em Saúde (S-RES). [Internet]. Sociedade Brasileira de Informática em Saúde [acesso em 18 out 2012]. 2011. Disponível: http://www. sbis.org.br/certificacao/Manual_Certificacao_SBIS_ CFM_2011_v4_Consulta_Publica.pdf

20. Kossman SP, Scheidenhelm SL. Nurses' perceptions of the impact of electronic health records on work and patient putcomes. Comput Inform Nurse. [Internet] 2008; 26(2). [acesso em 26 set 2012]. Disponível: http://journals.Iww.com/cinjournal/ abstract/2008/03000/nurses__perceptions_of_the_ impact_of_electronic.5.aspx

21. Moreno FN, Cubas MR, Malucelli A, Silva CL. Recurso computacional auxiliar ao ensino do raciocínio diagnóstico: intenções e valores identificados. Cogitare enferm. [Internet] 2013; 18(4). [acesso em 13 jan 2014]. Disponível: http://ojs.c3sl.ufpr.br/ojs/index.php/ cogitare/article/view/34918/21672

22. Malucelli A, Otemaier KR, Bonnet M, Cubas MR, Garcia TR. Sistema de informação para apoio à Sistematização da Assistência de Enfermagem. Rev. bras. enferm. [Internet] 2010; 63(4). [acesso em 13 jan 2014]. Disponível: http://dx.doi.org/10.1590/S003471672010000400020. 